\title{
3D Reconstruction of Weak Texture Surface Based On Binocular Vision
}

\author{
Fang Fan ${ }^{1}$, Zhenwei Wang ${ }^{2}$,Shuangyin Liu $^{3}$, Ye Tian ${ }^{3}$ \\ ${ }^{1,2,3}$ School of Astronautics and Aeronautics, University of Electronic Science \& Technology of China, \\ Chengdu, 611731, PR China. \\ ${ }^{1}$ 13007574791@163.com, ${ }^{2}$ wangjanvey@163.com
}

Keywords:binocular vision,key points,circular markers,3D reconstruction

Abstract-With the rapid development of computer science and image processing technology, 3D reconstruction and measurement of surface based on computer vision have become a research trends. However,for the weak texture surface,it is difficult to extract enough key points through general corner detection algorithms. Based on the principle of binocular stereo vision,this paper proposes a method of pasting circular markers to research 3D reconstruction of weak texture surface. This method can rich the surface's texture information used to feature extraction,so it can efficiently extract more key points. The results show that this proposed method is feasible and greatly improve $3 \mathrm{D}$ reconstruction of weak texture surface.

\section{Introduction}

A binocular vision system simultaneously captures two pictures of a scene using two cameras that are separated keeping a known distance. Then,the depth information of the scene can be obtained by parallax principle and triangle measurement principle.

In our paper,we take a turbine blade as the example of weak texture surface. As the key part of machinery, the distortion of the turbine blade is more and more obvious and it doesn't have enough texture information. Therefore,we can't get enough information to reconstruct by the general corner detection algorithms, such as Harris corners[1],SIFT features[2] and zero-crossing points[3]. For now,most researchers solve this problem using the grating projection method to rich texture information. Jia Qianqian designed and built the camera measurement system based on closed-light-section method[4]. Xu Hui and Zhang Jianlong realized 3D reconstruction of complex parts' surface based on surface structured light projection method[5]. However,the light projection method also has limitations due to noise interference,instability and complexity of this system.

To overcome the traditional problems of the light projection method,we propose to rich texture information of the turbine blade with pasting circular markers orderly. The proposed method can extract enough feature points by detecting center of circle using the principle of Circle Hough Transformation(CHT). The following sections will discuss stereo calibration,feature extraction,stereo match and $3 \mathrm{D}$ reconstruction.

\section{Stereo Calibration}

For a binocular vision system, there are two parts to finish the stereo calibration.

A.Single camera calibration. This part is to obtain intrinsic parameters,extrinsic parameters and distortion coefficients. It has been researched widely in [6,7].

B.Stereo calibration. Structure parameters which describe the relative position and rotation of two cameras are calibrated in this part.

For the convenience of calculation,this paper uses Camera Calibration Toolbox for Matlab. The 
calibration results are consistent with the reference [8] which was done using the method of reference [9] by our laboratory. The calibration results[8] are shown as follow:

$$
\begin{aligned}
& A_{1}=\left[\begin{array}{ccc}
875.4876 & 0 & 318.5514 \\
0 & 896.7750 & 174.7128 \\
0 & 0 & 1
\end{array}\right], \quad A_{r}=\left[\begin{array}{ccc}
855.14937 & 0 & 297.00109 \\
0 & 871.09273 & 155.25671 \\
0 & 0 & 1
\end{array}\right] \\
& R=\left[\begin{array}{ccc}
0.9998 & -0.0038 & 0.0197 \\
0.0034 & 0.9998 & 0.0189 \\
-0.0197 & -0.0189 & 0.9996
\end{array}\right], \quad T=\left[\begin{array}{llll}
-79.82416 & 1.86936 & -35.60430
\end{array}\right]
\end{aligned}
$$

Where, $A_{l}$ and $A_{\mathrm{r}}$ are the intrinsic parameters of the left camera and the right camera. $\mathrm{R}$ and $\mathrm{T}$ respectively express rotation matrix and translation matrix from the left camera to the right camera.

\section{Feature Extraction}

For the weak texture surface, extracting enough feature points is important for 3D reconstruction. Firstly, we need paste circular markers on the surface of the turbine blade. After acquiring 2D images of the turbine blade with pasted markers, detecting center of circle use the method of CHT. The principle of CHT is as follow.

We suppose that the equation of a circle show as in (1):

$$
(x-a)^{2}+(y-b)^{2}=r^{2}
$$

where $(\mathrm{a}, \mathrm{b})$ is the circle center,and $\mathrm{r}$ is the radius. $\left(x_{i}, y_{i}\right)(\mathrm{i}=1,2, \ldots, \mathrm{n})$ is a set of all points on the circumference of a circle.Then, in a-b-r parameter space,the equation of the set of $\left(x_{i}, y_{i}\right)$ is expressed as (2):

$$
\left(\mathrm{a}-x_{i}\right)^{2}+\left(b-y_{i}\right)^{2}=r^{2}
$$

Eq.2 is a 3-dimensional cone. Any point $\left(x_{i}, y_{i}\right)$ on the circumference in image space is corresponding to a 3-dimensional cone in the a-b-r parameter space. All points on a circumference will be a cluster of 3-dimensional cones. A circle in image space map into the parameter space is as shown in Fig.1.

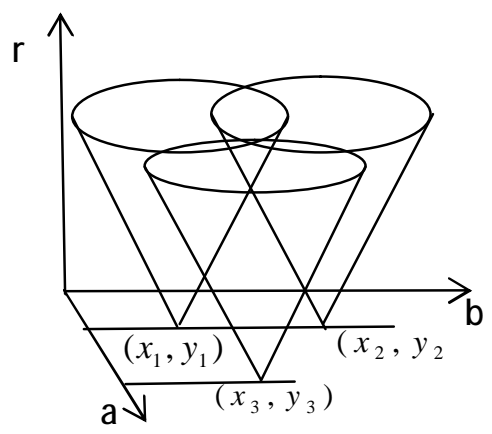

Fig.1 Circle in the a-b-r parameter space

If we want to detect the coordinate of the circle's center and the radius, we need build a 3-dimensional accumulator $\mathrm{A}(\mathrm{a}, \mathrm{b}, \mathrm{r})$ in the parameter space( $\mathrm{r}$ is a variate). According to Eq.2,(a,b,r) is calculated for each pixel point of the circle's circumference in image space. When there exists 
repetitive intersections in a same position, the value of $\mathrm{A}(\mathrm{a}, \mathrm{b}, \mathrm{r})$ add one at this position. When the value of the accumulator reaches a maximum in a position,this point at this position is regarded as the circle center.

In this paper,the acquired image should be preprocessed by Gaussian Filter before employing CHT. However,there are few undesired circle detected and some desired circle undetected. Hence,we need to further process the image by deleting undesired points and adding the missing points by partial detection. Figure 2 shows the image after this processing method.

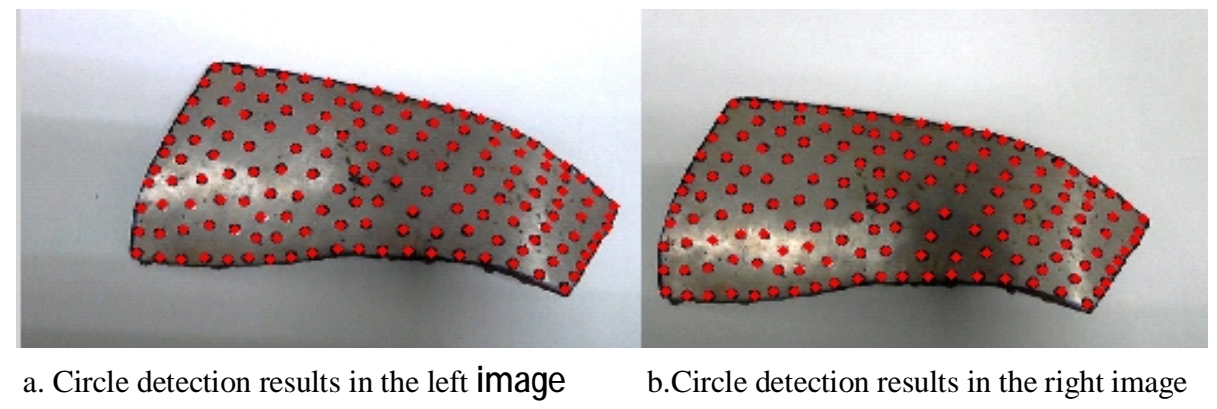

Fig.2 Circle detection results after further processing

\section{Stereo Match}

We have extracted enough feature points based on the method we proposed in last section. Then,the points in the left image and in the right image are matched using correlation matching,uniqueness constrain,parallax smoothness constraint and the epipolar constraint[8]. Figure 3 shows the matching results making the matched points with the same number.

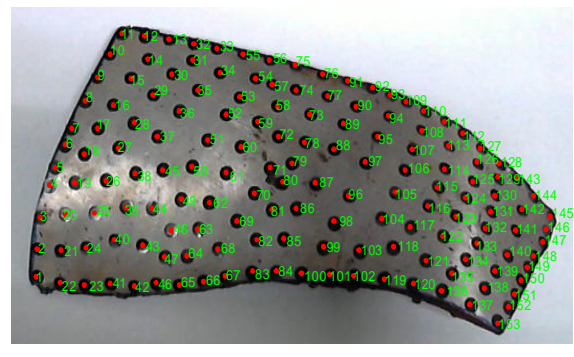

a. The left image

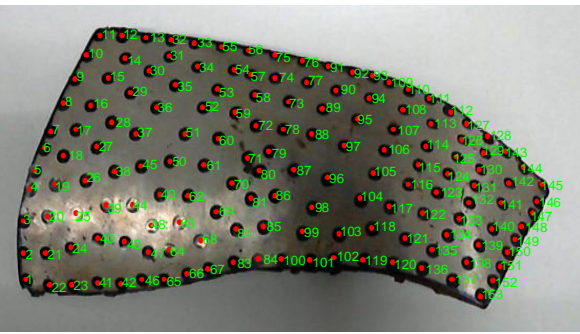

b. The right image

Fig.3 Results of points matching

\section{D reconstruction}

On the base of getting intrinsic parameters and structure parameters of the two cameras, we could calculate the spatial coordinates of matched points using parallax principle and triangulation. Then,these spatial points are used to surface fitting based on the NURBS method[10] as control points. To make the surface more smooth,we employ the interpolation method for the fitted surface.Figure 4 shows the final fitting results. Table 1 shows the measurement results of the reconstructed turbine blade. This results indicates that the measurement values are close to the real values. Hence,this proposed method is feasible. 
Table 1. Measured Results

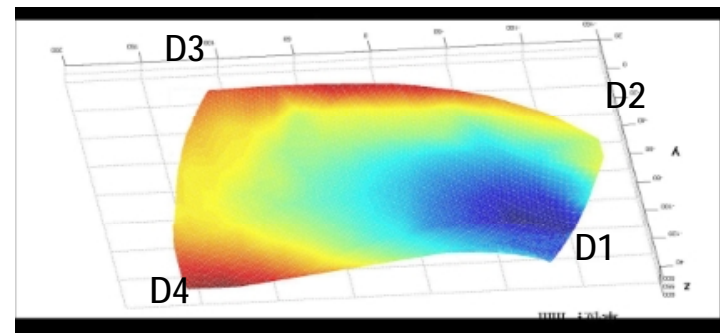

\begin{tabular}{|c|c|c|}
\hline $\begin{array}{c}\text { Distance } \\
{[\mathrm{mm}]}\end{array}$ & $\begin{array}{c}\text { Real } \\
\text { Length }\end{array}$ & $\begin{array}{c}\text { Measured } \\
\text { Length }\end{array}$ \\
\hline D1D2 & 150.4 & 150.26 \\
\hline D2D3 & 270.4 & 279.62 \\
\hline D3D4 & 91.3 & 91.67 \\
\hline D4D1 & 289.5 & 285.83 \\
\hline
\end{tabular}

Fig.4 3D Reconstruction results for the rotor blade after interpolation

\section{Conclusion}

In this paper,we finish 3D reconstruction of weak texture surface using the method of pasting circular markers based on binocular vision. Feature extraction based on pasting circular markers are described in detail. Most circle centers are successfully extracted with the CHT method. The reconstruction result shows that the proposed method is reasonable. However,we cannot extract all the points on the first operation and some points are detected error. To improve the accuracy of circle detection will be our next work.

\section{Acknowledgements}

This work was financially supported by the Fundamental Research Funds for the Central Universities (No.ZYGX2014J095) and the National Natural Science Foundation of China (No.51005040)

\section{Reference}

[1] C.Haaris and M.Stephens: A combined corner and edge detector, Proc. 4th Alvey Vision Conf., pp. 147-151, 1988.

[2] D. G. Lowe: Object recognition from local scale-invariant features, Proc. 7th IEEE Int'l Conf.on Computer Vision (ICCV'99), pp. 1150-1157, Corfu, Greece, 1999.

[3] W. Eric and L. Grimson:Computational experiments with a feature based stereo algorithm, IEEE Trans. Pattern Anal. Mach Intell., vol. 7,no. 1, pp. 17-34, 1985.

[4] Jia Qianqian,Zhao Bohua: 3D Surface Measurement System for Free-Form Surface Based On Closed-Light-Section Method,Chinese Journal of Scientific Intrument, Vol.30,No.6,Jun.2009

[5] Xu Hui,Zhang Jianlong,Liu Jingnan and UCHIDA Yoshihisa: Research On the Part Surface Measurement and 3D Reconstruction,Journal of NanJing Normal University,Vol.11,No.2,Jun.2011

[6] Sun J, Zheng N, Shun H Y. Stereo matching using belief propagationg [J]. IEEE Transactions on Pattern Analysis and Machine Intelligence, 2003, 25(7):787-800.

[7] Simith A R.,Planar.: 2-Pass Texture Mapping and Warping[J].,Computer Graphics, 1987,21(4):263-272

[8] Zhenwei WANG,Fang FAN and Shuangyin LIU: Measurement method for complex shape of hydraulic turbine blade using binocular vision,CMSA 2015 International Conference,p:344-348

[9] Z.Y ZHANG: A Flexible New Technique for Camera Calibration,IEEE Transactions on Pattern Analysis and Machine Intelligence. 22(11)(2000) 1330-1334

[10] He Yuanjun: Computer graphics[M], Beijing Institute of Machinery,2006 